\title{
Research on Finger Vein Acquisition Based on Wavelength Choice
}

\author{
Desen Yin ${ }^{1,2, a}$, Zengxi Ding ${ }^{1,2, b}$ \\ ${ }^{1}$ The First Research Institute of the Ministry of Public Security of People's \\ Republic of China \\ ${ }^{2}$ Key Laboratory of Documents Anti-counterfeiting, Ministry of Public \\ Security, People's \\ Republic of China \\ ayindesen@aliyun.com, ${ }^{b}$ zengxiding@163.com
}

\begin{abstract}
Finger vein biometric is a novel technology for personal identification, and acquisition of finger vein image is difficult in finger vein recognition. In this paper, scattering and absorption characteristics of skin, muscle, phalanx and vein of finger under near infrared light are analyzed, and the mathematical model about imaging process of finger vein is established. Then on this basis, the relation between light source wavelength and image quality is investigated, and images quality under two single wavelengths including $850 \mathrm{~nm}$ and $940 \mathrm{~nm}$ are analyzed by use of image contrast. Experimental results show that the mathematical model is available to imaging process of finger vein.

Keywords: finger vein acquistition, tissue optical, Image quality, near infrared light
\end{abstract}

\section{Introduction}

Finger vein recognition is a novel type of biometrics, which take distribution patterns of finger vein apply in identity authentication. Researchers have determined that the pattern of finger vein is unique to a specific individual, difficult to forge, contact-less, not affected by race and skin discolorations, the technology has a well applying prospect and developmental potential [1]. The principle of finger vein identification is that the infrared light with specific wavelength can pass through most of the human tissues while the hemoglobin in the blood can absorb the infrared light fully. When the finger is put in opening 
concave, the near-infrared light source becomes parallel light through lens and irradiates the detecting position of finger directly, the infrared light through vein is absorbed by hemoglobin so that the vein structure image passes the light filter and is ingested by the image ingesting part. In the system, an infrared light is transmitted from the back-side of a finger. A finger is placed between the infrared light source and a camera. As hemoglobin in the blood absorbs the infrared light, the finger vein patterns spread in the palm-side of a hand are captured as shadows $[2,3]$. In the process of imaging, there are many differences on quality of finger vein image because of the wavelength choice and the differences between individual fingers. The poor quality image will lose many of useful information, and which can bring the difficulty to feature extraction and identity recognition. It is very important to choice a proper wavelength of light source, so this paper analyzes the optical characteristics of different finger tissue, and designs a mathematical model of finger vein imaging according to relationship between light wavelength and the image quality of finger vein. The experimental results show that the mathematical model is effective for imaging of finger vein.

\section{Spectrum analysis}

In the range of electromagnetic waves, the near infrared light can propagate deeply inside the human tissue, especially for near infrared light with wavelength of $700 \mathrm{~nm}-1300 \mathrm{~nm}$, the sepicial range also referred to as tissue optical window, because the absorption and scattering to light within the range are minimal. In the band of near infrared light, scattering is the most important when the light propagate in human tissue, which can lead to rapid diffusion of light energy. However effect of scattering has little change with wavelength of light, the effective range of the tissue optical window is mainly limited by absorption characteristics of human tissue. To imaging technology of finger vein, it is significant to improve image quality and penetration depth through selecting proper light source in the tissue optical window. Generally, major tissue of human finger includes the skin, muscle and phalanx, the optical properties of these tissues will be described as follow.

1) Skin. There are three layers in finger skin, namely epidermis, dermis and subcutaneous tissue. Epidermis layer is very thin, which is composed of horny layer, transparent layer, granular layer, spinous layer and the substrate layer. The first four layers have weak ability in absorption to near infrared light, and most light can penetrate into the lower layers of the skin. The substrate layer contains melanin granules, responsible for manufacturing melanin which can absorb a part 
of light. The quantity of absorption can be neglected when considering the less content of melanin in skin of finger. The dermis layer is composed of papillary layer and reticular layer, the main structure is the elastic fibers and connective tissue, and contains a lot of water, the layer can absorb part of near infrared light. The subcutaneous tissue consists of fat cell and vascular network which is made up of a lot of vein. Water, hemoglobin and cytochrome in the skin play a major role in absorption of light. In the range of the tissue optical window, absorption coefficient of bilirubin, deoxygenated hemoglobin and oxyhemoglobin is too small to measure. According to whether combined with oxygen, Hemoglobin is divided into deoxygenated hemoglobin and oxyhemoglobin. According to the relevant literature, absorption coefficient of water is far less than that of deoxygenated hemoglobin in the range of $600 \mathrm{~nm}-800 \mathrm{~nm}$. In the range of $800 \mathrm{~nm}$ - 1000nm, absorption coefficient of water increases rapidly, and an absorption peak is located at the wavelength of near $970 \mathrm{~nm}$, at the same time, in the range of $600 \mathrm{~nm}-1000 \mathrm{~nm}$, the absorption coefficient of fat is larger and its effect for light absorption cannot be ignored.

2) Phalanx and muscle. There are fourteen bones in human hand, thumb has two bones and others have three, namely the proximal phalanx, middle phalanx and distal phalanx. Each phalanx is divided into bottom, body and head. There is a few data on absorption properties of phalanx to near infrared light. But many data on absorption properties of skull to near infrared light can be found. According to relevant data, the skull has absorption effect to near infrared light, but the change of absorption coefficient with wavelength is not obvious. Therefore, the effect of phalanx on a finger vein imaging can be ignored. Finger muscle's absorption to near infrared light is mainly due to the presence of myoglobin, which is found in muscle cells, it is red pigment combining with oxygen, and having the same absorption spectrum as the hemoglobin in red blood cells. However, myoglobin is not so easy to be oxidized. At the same time, the finger muscle is internal tissue where light propagates belong to the strong scattering. Finger muscle has little effect on finger vein imaging.

Based on above analysis, absorption characteristics of finger tissue to near infrared light are divided into three kinds:

Firstly, phalanx has effect to near infrared light, but the effect has nothing to do with change of light wavelength, so the effect of phalanx can be ignored in the spectral analysis of finger vein imaging. For muscle in the finger, because absorption coefficient is little, the influence to finger vein imaging in different wavelength is also negligible because of strong scattering propagation effect.

Secondly, distribution of water and fat are relatively uniform in every finger, 
and absorption characteristics that water and fat to near infrared light is same, the absorption effect is smaller when the wavelength is smaller, but absorption will increase quickly when wavelength of light increase to a certain extent, accordingly, finger vein image will overall darken under the same light intensity.

Thirdly, Finger skin plays a key role in imaging process of finger vein, whether in the transmission imaging or reflective imaging, before coming out from finger, light energy should be uniform because of the strong scattering effect that finger tissue to infrared light. After light passes through the finger by style of transmission or reflection, the light intensity will become uneven because of the absorption by hemoglobin in finger vein when the light passes through skin. When image sensor receives the uneven light, a finger vein image is formed.

\section{Mathematical model}

It is assumed that the energy of incident light is $E_{i}$, due to imaging of finger vein is superficial venous angiography, energy of light in finger can be divided into three parts: $E_{a}$ is the energy that absorbed by finger tissue, $E_{o}{ }^{\prime}$ is the energy that will transmit out from finger after strong scattering, $E_{r}{ }^{\prime}$ is the energy that will return to the incident space after strong scattering. Because of the strong scattering effect of the finger tissues, $E_{o}{ }^{\prime}$ and $E_{r}{ }^{\prime}$ can be regarded as uniform, and when this two part of the light energy transmit through the finger skin, they will show the shadow of vein because of absorption by surface finger vein. $E_{o}$ is the light energy of transmission imaging, and $E_{r}{ }^{\prime}$ is the light energy of reflection imaging. The above procedure is described in Fig.1, and the relationship between the parts of the light energy is

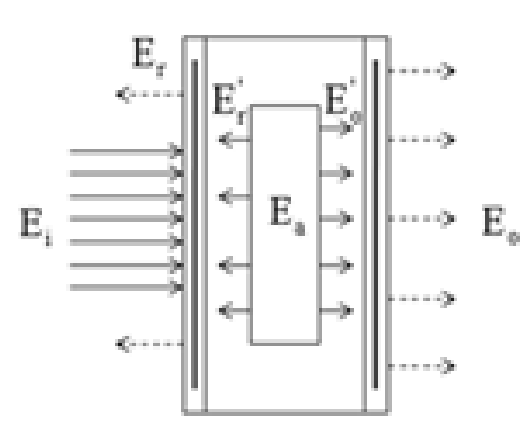


Fig.1 Mathematical model

shown in Eq.1, Eq.2 and Eq.3.

$$
\begin{gathered}
E_{i}=E_{a}+E_{r}^{\prime}+E_{o}^{\prime}=E_{a}+E_{s} \\
\Delta E_{r}=E_{r}^{\prime}-E_{r}=F\left(u_{a}\right) \cdot E_{r}^{\prime} \\
\Delta E_{o}=E_{o}^{\prime}-E_{o}=F\left(u_{a}\right) \cdot E_{o}^{\prime}
\end{gathered}
$$

While, $u_{a}$ is absorption coefficient of finger vein to near infrared, $F\left(u_{a}\right)$ is absorption correlation function of finger vein to near-infrared.

According to the $E q .1$, the more $E_{a}, E_{s}$ is smaller, i.e. the more energy absorbed by finger tissue, incident light energy of refection and transmission imaging system is smaller, when $u_{a}$ is constant, $\Delta E_{r}$ and $\Delta E_{o}$ will become smaller, image contrast of finger vein will reduce too. Therefore, when intensity of incident light is constant, the more light energy absorbed by finger, the intensity of transmission and reflection light is smaller. According to the Eq.3, if other absorption coefficient has little change, finger vein image contrast will reduce accordingly.

\section{Experimental analysis}

In order to verify the validity of mathematical model, we use 850nm and 940nm infrared light to capture finger vein image. In process of image acquisition, intensities of two kinds of light are adjusted to make a similar visual effect of finger vein image. In this paper, finger vein image is divided into background area and vascular area, since the brightness of background depends on the intensity of transmitted light, pixel gray in background area can be used as the parameters described the light intensity. At the same time, the difference of pixel gray between background area and the vascular area can be used as the parameters of image contrast. The experimental steps are as follows:

1) Images of finger vein are captured from 2 fingers using $850 \mathrm{~nm}$ and 940nm light source, the number of images is 5 per finger. Images of finger vein captured by $850 \mathrm{~nm}$ are shown in Fig.2 (a) and (b), images captured by 940nm are shown in Fig.3 (a) and (b).

2) Binary image can be got through segmentation algorithm, and the binary images are shown in Fig.2 (c), Fig.2 (d), Fig.3 (c) and Fig.3 (d). For binary image, the white area is vascular region of finger vein and the black area is background region.

3) Based on original and binary image of finger vein, mean values of pixel gray 
in vascular area of original images are calculated, and mean values of pixel gray in background area of original images are calculated too.

4) Mean value of pixel gray in background area is taken as the parameter of light source intensity, and difference of mean value between background area and vascular area is taken as the parameter of image contrast. Statistical data are showed in Table 1, 2, 3, 4.

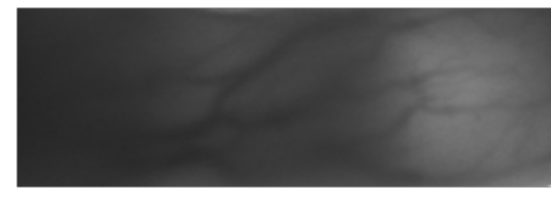

(a)Original image of the first finger

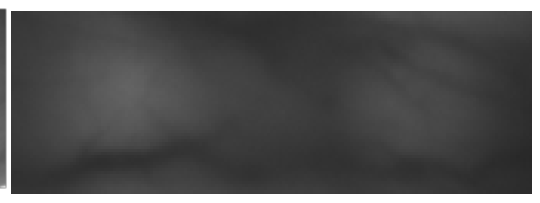

(b)Original image of the second finger

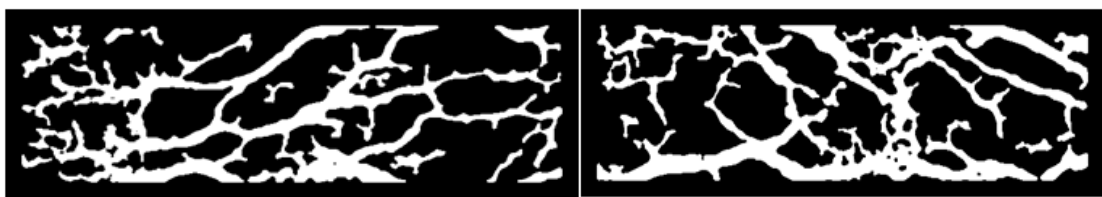

(c)Binary image of the first finger

(d)Binary image of the second finger

Fig.2 Images acquired under 850nm. 


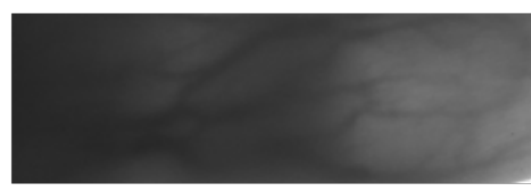

(a)Original image of the first finger

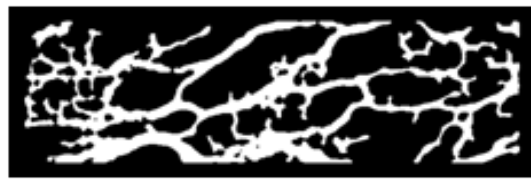

(c)Binary image of the first finger

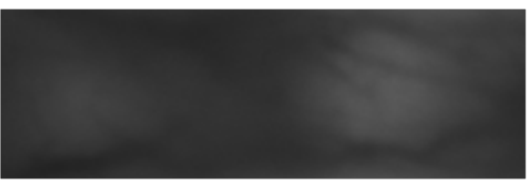

(b) Original image of the second finger

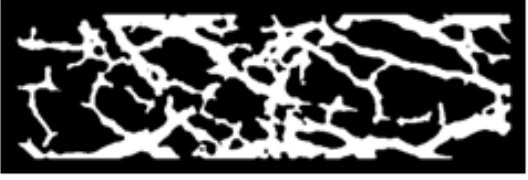

(d) Binary image of the second finger

Fig.3 Images acquired under 940nm.

Table 1: Light intensity of the first finger.

\begin{tabular}{|l|l|l|l|l|l|l|}
\hline \multicolumn{3}{|c|}{ Wavelength } & \multicolumn{3}{c|}{ Experimental data } & \multicolumn{2}{c|}{ Mean } \\
\hline 940 & 75.52 & 85.11 & 72.00 & 71.99 & 79.13 & 76.75 \\
\hline 850 & 71.97 & 72.57 & 70.09 & 70.95 & 69.12 & 70.94 \\
\hline
\end{tabular}

Table 2: Image contrast of the first finger.

\begin{tabular}{|l|l|l|l|l|l|l|}
\hline \multicolumn{3}{|c|}{ Wavelength } & \multicolumn{3}{c|}{ Experimental data } & \multicolumn{2}{c|}{ Mean } \\
\hline 940 & 9.77 & 9.67 & 8.90 & 8.13 & 6.72 & 8.63 \\
\hline 850 & 8.32 & 9.49 & 8.01 & 8.28 & 8.26 & 8.47 \\
\hline
\end{tabular}

Table 3: Light intensity of the second finger.

\begin{tabular}{|l|l|l|l|l|l|l|}
\hline \multicolumn{3}{|c|}{ Wavelength } & \multicolumn{3}{c|}{ Experimental data } & \multicolumn{2}{c|}{ Mean } \\
\hline 940 & 64.38 & 65.50 & 70.82 & 68.28 & 67.48 & 67.29 \\
\hline 850 & 57.30 & 59.45 & 60.99 & 60.90 & 56.04 & 58.93 \\
\hline
\end{tabular}

Table 4:Image contrast of the second finger.

\begin{tabular}{|l|l|l|l|l|l|l|}
\hline \multicolumn{3}{|c|}{ Wavelength } & \multicolumn{3}{c|}{ Experimental data } & \multicolumn{2}{c|}{ Mean } \\
\hline 940 & 5.89 & 7.31 & 8.77 & 7.76 & 7.55 & 7.45 \\
\hline 850 & 4.43 & 8.55 & 9.24 & 8.69 & 7.34 & 7.65 \\
\hline
\end{tabular}

According to the data in Table 1 and Table 2, parameters of image contrast corresponding to the first finger in $940 \mathrm{~nm}$ and $850 \mathrm{~nm}$ are 8.63 and 8.47 respectively, and parameters of light intensity are 76.75 and 70.94 respectively. Parameters of image contrast corresponding to the second finger in $940 \mathrm{~nm}$ and 
850nm are 7.45 and 7.65 respectively, and parameters of light intensity are 67.29 and 58.93 respectively. It can be seen from above data: In order to achieve the same image contrast, light intensity of $940 \mathrm{~nm}$ must be greater than that of $850 \mathrm{~nm}$. In premise of light intensity is not different, image contrast of $940 \mathrm{~nm}$ imaging system is lower than that of $850 \mathrm{~nm}$ imaging system, to improve the image contrast, the light intensity of imaging system should be enhanced. The experimental result is same with the conclusion of mathematical model that be introduced in Section 3.

\section{Conclusion}

Based on the analysis about absorption and scattering characteristics of the finger tissue to infrared light, such as muscle, bones and skin, the mathematical model of finger vein imaging was given, and the relationship between wavelength of light source and image quality of finger vein was presented by this mathematical model. Meanwhile, mean value of pixel gray in background area are taken as the parameter of light intensity, difference of mean value between background area and vascular area are taken as representation of image contrast, and finger vein image acquired under $850 \mathrm{~nm}$ and $940 \mathrm{~nm}$ are analyzed experimentally. Experimental results proved that it must to raising the brightness of $940 \mathrm{~nm}$ light source in order to achieve the same image contrast with $850 \mathrm{~nm}$ light source, which verify the validity of the mathematical model of finger vein imaging.

\section{References}

[1] John D, Nicholas M, Peter T. Biometrics: Identity Assurance in the Information Age.Berkeley, California: Osborne, 2003.

[2] JI Zijuan, Bao Jiaqi. An Introduction of Optical System in Finger Vein Verification System. J. Journal of Hubei Institute of Education. 24(2) 40-42, 2007.

[3] Yanggan Dai, Beining Huang. A Method for Capturing the Finger vein Image Using Nonuniform Intensity Infrared Light. 2008 Congress on Image and Signal Processing, 501-505, 2008.

[4] Yuan Weiqi, Yang Guotian, Li Wei. Research on palm vein acquisition system based on wavelength choice. J. LASER \& INFRARED. 41(2) 234-239, 2011. 
[5] Li Wei, Yuan Weiqi. Imaging Quality Analysis on Palm Vein under Different Wavelengths Near-IR. J. Computer Engineering and Applications. 47(30) 15-18, 2011. 